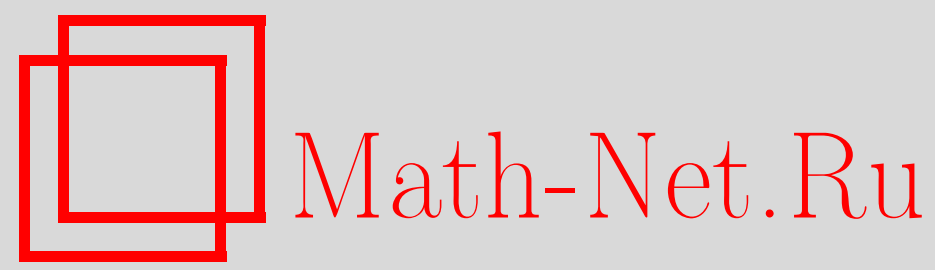

А. В. Иванов, Асимптотически оптимальные критерии в задаче различения параметрических гипотез о распределении случайного вектора. II, Матем. вопр. криптогр., 2015, том 6, выпуск 4, 49-64

DOI: https://doi.org/10.4213/mvk167

Использование Общероссийского математического портала Math-Net.Ru подразумевает, что вы прочитали и согласны с пользовательским соглашением

http://www . mathnet.ru/rus/agreement

Параметры загрузки:

IP: 54.198 .187 .58

26 апреля 2023 г., 10:32:50

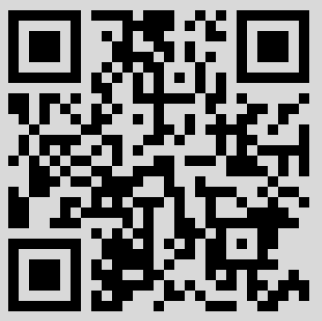


УДК: 519.233 .3

\title{
Асимптотически оптимальные критерии в задаче различения параметрических гипотез о распределении случайного вектора. II
}

\author{
А. В. Иванов \\ Национальный исследовательский университет «Высшая школа экономики», \\ Москва \\ Получено 20.IV.2015
}

Построены асимптотически оптимальные критерии в задаче различения простых гипотез о параметрах комбинированного полунепрерывного канала связи в схеме серий для случая векторного параметра, характеризующего распределение дискретной помехи. Найдены вероятностные характеристики критериев (в частности, асимптотически минимальный объем выборки при заданных вероятностях ошибок первого и второго рода). Показано, что минимальный объем выборки существенно зависит от соотношений между параметрами канала.

Ключевые слова: асимптотически оптимальный критерий, комбинированный полунепрерывный канал связи, схема серий, асимптотически минимальный объем выборки

Asymptotically optimal criteria for testing hypotheses on the distribution of a random vector. II

\section{A. V. Ivanov \\ National Research University "Higher School of Economics", Moscow}

\begin{abstract}
Asymptotically optimum criteria for testing two simple hypotheses on the vector parameters of the distribution of discrete noise in the combined semicontinuous communication channel are constructed. A probabilistic characteristics of these criteria (in particular, asymptotically minimal sample size guaranteeing the given values of error probabilities of two kinds) are obtained. It is shown that the minimal sample size depends on the relations between the channel patameters.
\end{abstract}

Key words: asymptotically optimal criterion, combined semicontinuous communication channel, asymptotically minimal sample size

Citation: Mathematical Aspects of Cryptography, 2015, vol. 6, no. 4, pp. 49-64 (Russian). 
А. В. Иванов

\section{1. Введение}

Рассматривается модель комбинированного полунепрерывного канала связи (КПКС), представляющего собой комбинацию двоичного симметричного канала и непрерывного аддитивного шума (см., например, [3, глава 7]). Каждый КПКС однозначно определяется заданием набора параметров. Целью настоящей статьи является построение асимптотически оптимальных критериев различения двух простых гипотез о параметрах КПКС в схеме серий для векторного параметра дискретной помехи.

Определим формально модель КПКС. Пусть $B=\{0,1\}$. Рассмотрим последовательность независимых случайных векторов

$$
X^{(t)}=\left(X_{1}^{(t)}, X_{2}^{(t)}, \ldots, X_{m}^{(t)}\right) \in B^{m}, B^{m}=\{0,1\}^{m}, t \in \mathbb{Z}^{+}=\{1,2, \ldots\},
$$

с независимыми одинаково распределенными координатами, $\mathbf{P}\left(X_{j}^{(t)}=\tau\right)=$ $=2^{-1}, \tau \in B, j=1,2, \ldots, m$. Везде в дальнейшем предполагается, что $m \in$ $\in \mathbb{Z}^{+}=\{1,2, \ldots\}$, фиксировано и конечно.

Пусть $\varepsilon^{(t)}=\left(\varepsilon_{1}^{(t)}, \varepsilon_{2}^{(t)}, \ldots, \varepsilon_{m}^{(t)}\right) \in B^{m}-$ последовательность не зависящих от последовательности $\left\{X^{(t)}\right\}_{t=1}^{\infty}$ независимых случайных векторов с независимыми координатами,

$$
\mathbf{P}\left(\varepsilon_{j}^{(t)}=\tau\right)=\frac{1+(-1)^{\tau} \delta_{j}}{2}, \tau \in B, \quad\left|\delta_{j}\right| \leq 1, j=1,2, \ldots, m .
$$

Вектор $\delta=\left(\delta_{1}, \delta_{2}, \ldots, \delta_{m}\right)$ является параметром двоичного симметричного канала.

Рассмотрим последовательность случайных величин

$$
\eta_{t}^{*}=f\left(X^{(t)} \oplus \varepsilon^{(t)}\right)+\xi_{*}^{(t)},
$$

где $\left\{\xi_{*}^{(t)}\right\}_{t=1}^{\infty}$ - последовательность независимых одинаково распределенных случайных величин с абсолютно непрерывным распределением, не зависящих от векторов $\left\{X^{(t)}, \varepsilon^{(t)}\right\}_{t=1}^{\infty}$, для которых существуют и конечны $\mathbf{E} \xi_{*}^{(t)}=$ $=\mu, \mathbf{D} \xi_{*}^{(t)}=\sigma^{2}>0 ; \oplus-$ операция покоординатного сложения векторов по модулю $2 ; f: B^{m} \rightarrow R-$ заданная псевдобулева функция.

Введем в рассмотрение последовательность случайных величин

$$
\eta_{t}=\frac{\eta_{t}^{*}-\mu}{\sigma}=\lambda f\left(X^{(t)} \oplus \varepsilon^{(t)}\right)+\xi^{(t)},
$$


где $\left\{\xi^{(t)}\right\}_{t=1}^{\infty}-$ последовательность независимых одинаково распределенных случайных величин с абсолютно непрерывным распределением $\mathbf{E} \xi^{(t)}=0$, $\mathbf{D} \xi^{(t)}=1, \lambda=\sigma^{-1}-$ параметр аддитивной помехи.

При заданных функции $f$, векторе $\delta=\left(\delta_{1}, \delta_{2}, \ldots, \delta_{m}\right)$ и параметре $\lambda=\sigma^{-1}$ модель (1) определена однозначно.

Далее будем использовать следующие обозначения: $g(z)-$ плотность, $G(z)=\int_{-\infty}^{z} g(u) d u, z \in \mathrm{R},-$ функция распределения случайной величины $\xi^{(t)} ; \phi(z)=\frac{1}{\sqrt{2 \pi}} e^{-\frac{z^{2}}{2}}-$ плотность, $\boldsymbol{\Phi}(z)=\int_{-\infty}^{z} \phi(u) d u, z \in \mathrm{R},-$ функция распределения, $\zeta_{\alpha}-$ квантиль стандартного нормального распределения $\mathcal{N}(0 ; 1)$ уровня $\alpha \in(0,1)$.

Предположим, что имеется выборка, состоящая из независимых одинаково распределенных случайных векторов

$$
\left\{\left(X^{(t)}, \eta_{t}\right)=\left(X^{(t)}, \lambda f\left(X^{(t)} \oplus \varepsilon^{(t)}\right)+\xi^{(t)}\right)\right\}_{t=1}^{\infty}
$$

Обозначим через $H_{f, \lambda, \delta}$ гипотезу, которая однозначно задается функцией $f$, векторным параметром $\delta=\left(\delta_{1}, \delta_{2}, \ldots, \delta_{m}\right)$ и параметром $\lambda$.

Пусть $H_{f, \lambda, \delta}, H_{f^{*}, \lambda^{*}, \delta^{*}}$ - две простые гипотезы, для которых выполнено хотя бы одно из условий:

1) существует такое $x \in B^{m}$, что $f(x) \neq f^{*}(x)$;

2) существует такое $j \in\{1,2, \ldots, m\}$, что $\delta_{j} \neq \delta_{j}^{*}$;

3) $\lambda \neq \lambda^{*}$.

При выполнении хотя бы одного из условий 1-3 требуется построить асимптотически оптимальный критерий различения гипотез $H_{f, \lambda, \delta}, H_{f^{*}, \lambda^{*}, \delta^{*}}$ по выборке (2) в схеме серий при различных соотношениях между параметрами КПКС.

На протяжении настоящей работы для упрощения обозначений некоторые индексы в обозначении гипотез $H_{f, \lambda, \delta}$ (в зависимости от конкретной пары различаемых гипотез) там, где это не вызовет разночтений, будут опускаться: например, если речь идет о построении критерия различения гипотез $H_{f, \lambda, \delta}$ и $H_{f, \lambda, \delta^{*}}$, то будем обозначать соответствующие гипотезы через $H_{\delta}$ и $H_{\delta^{*}}$. Кроме того, через $H^{(0)}$ далее обозначается гипотеза $H_{f, 0, \delta}$, которая будет рассматриваться в качестве вспомогательной. Также считаем, что $\lambda=\lambda^{*}$ при $\lambda \neq 0$, поэтому в дальнейшем фактически будут различаться гипотезы $H_{f, \delta}$ и $H_{f^{*}, \delta^{*}}$.

Результаты настоящей статьи представляют собой обобщение ранее рассмотренного автором случая скалярного параметра $\delta_{j} \equiv \delta, j=1,2, \ldots, m$ (см. [6-8, 10]). Некоторые результаты указанных работ могут быть получены в качестве следствий приведенных ниже теорем. Результаты для случая 
многомерного параметра $\delta$ ранее были частично приведены автором в тезиcax [9].

\section{2. Предварительные результаты}

Условимся в дальнейшем под записью $\delta=$ const подразумевать вектор с координатами $\delta=\left(\delta_{1}, \delta_{2}, \ldots, \delta_{m}\right)$, где $\delta_{j}=$ const, $j=1,2, \ldots, m$. В частности, через $\delta=a, a \in \mathrm{R}$, обозначим вектор $\delta=(a, a, \ldots, a)$. Запись $\delta=\delta^{*}$ будет обозначать, что $\delta_{j}=\delta_{j}^{*}, j=1,2, \ldots, m$. Если существует такое $j=$ $=1,2, \ldots, m$, что $\delta_{j} \neq \delta_{j}^{*}$, то будем писать $\delta \neq \delta^{*}$.

Пусть дана последовательность случайных векторов (2), где $X^{(t)}, \varepsilon^{(t)}$, $\xi^{(t)}, t=1,2, \ldots,-$ независимые в совокупности случайные векторы и случайные величины, имеющие следующие распределения:

$$
\begin{gathered}
\mathbf{P}\left(X^{(t)}=x\right)=2^{-m}, \\
\mathbf{P}\left(\varepsilon^{(t)}=u\right)=\pi_{\delta}(u)=2^{-m} \prod_{j=1}^{m}\left(1+(-1)^{u_{j}} \delta_{j}\right), \pi_{0}(u)=2^{-m}, x, u \in B^{m}, \\
\mathbf{P}\left(\xi^{(t)} \leq y\right)=G(y)=\int_{-\infty}^{y} g(v) d v, y \in \Re .
\end{gathered}
$$

Здесь $\pi_{\delta}(u)$ и $g(v)$ - плотности распределений вероятностей случайного вектора $\varepsilon^{(t)}$ и случайной величины $\xi^{(t)}$ по считающей и непрерывной доминирующим мерам соответственно, $\delta=\left(\delta_{1}, \delta_{2}, \ldots, \delta_{m}\right),\left|\delta_{j}\right| \leq 1, j=$ $=1,2, \ldots, m,-$ векторный параметр распределения случайного вектора $\varepsilon^{(t)}$.

Будем говорить, что справедлива гипотеза $H_{f, \lambda, \delta}$, если распределение случайной величины $\eta_{t}$ задается функцией $f$ и параметрами $\lambda, \delta$ модели (1), где $\delta=\left(\delta_{1}, \delta_{2}, \ldots, \delta_{m}\right)$.

Пусть $n \rightarrow \infty, \lambda \equiv \lambda(n) \rightarrow 0$ и сформулированы две простые гипотезы относительно параметров $f$ и $\delta$ :

$$
\begin{gathered}
H_{\delta}: f, \delta=\left(\delta_{1}, \delta_{2}, \ldots, \delta_{m}\right), \\
H_{\delta^{*}}: \quad f^{*}, \delta^{*}=\left(\delta_{1}^{*}, \delta_{2}^{*}, \ldots, \delta_{m}^{*}\right),
\end{gathered}
$$

где $\delta=$ const, $\delta^{*}=$ const и выполнено хотя бы одно из условий:

1) существует такое $x \in B^{m}$, что $f(x) \neq f^{*}(x)$;

2) существует такое $j \in\{1,2, \ldots, m\}$, что $\delta_{j} \neq \delta_{j}^{*}$ (в частности, пусть существует такое $i=1,2, \ldots, m: \delta_{i}>0$, но $\left.\delta_{j}^{*}=0 \forall j=1,2, \ldots, m\right)$.

Требуется построить асимптотически оптимальный критерий различения гипотез $H_{f, \delta}, H_{f^{*}, \delta^{*}}$. 
Рассмотрим статистику критерия различения гипотез $H_{f, \delta}$ и $H_{f^{*}, \delta^{*}}$, основанную на логарифме отношения правдоподобия. Обозначим через $p_{f, \lambda, \delta}(x, z)=\mathbf{P}\left(X^{(t)}=x, \eta_{t} \leq z\right)$ плотность распределения вектора $\left(X^{(t)}, \eta_{t}\right)$ из (2) по соответствующей доминирующей мере, через $p(x, z)=2^{-m} g(z)-$ плотность распределения вектора $\left(X^{(t)}, \eta_{t}\right)$ при $\lambda=0, \delta=0$. Далее через $\mathbf{E}_{\delta}($ ) обозначается соответствующее математическое ожидание по распределению, определяемому параметром $\delta$. Из разложения Тейлора следует, что при $n \rightarrow \infty, \lambda=\lambda(n) \rightarrow 0$

$$
\begin{gathered}
r_{f, \lambda, \delta}(x, z)=\frac{p_{f, \lambda, \delta}(x, z)}{p(x, z)}=\mathbf{E}_{\delta} \frac{g(z-\lambda f(x \oplus \varepsilon))}{g(z)}= \\
=\mathbf{E}_{\delta}\left[\frac{g(z)-\lambda g^{\prime}(z) f(x \oplus \varepsilon)+\frac{\lambda^{2}}{2} g^{\prime \prime}(z) f^{2}(x \oplus \varepsilon)+O\left(\lambda^{3}\right)}{g(z)}\right]= \\
=1-\lambda \frac{g^{\prime}(z)}{g(z)} \mathbf{E}_{\delta} f(x \oplus \varepsilon)+\frac{\lambda^{2}}{2} \frac{g^{\prime \prime}(z)}{g(z)} \mathbf{E}_{\delta} f^{2}(x \oplus \varepsilon)+O\left(\lambda^{3}\right) .
\end{gathered}
$$

Лемма 1. Пусть

$$
\begin{aligned}
a(z) & =\frac{g^{\prime}(z)}{g(z)}, & b(z) & =\frac{g^{\prime \prime}(z)}{g(z)}, \\
A_{f, \delta}(x) & =\mathbf{E}_{\delta} f(x \oplus \varepsilon), & B_{f, \delta}(x) & =\mathbf{E}_{\delta} f^{2}(x \oplus \varepsilon) .
\end{aligned}
$$

Тогда

$$
r_{f, \lambda, \delta}(x, z)=1-\lambda a(z) A_{f, \delta}(x)+\frac{\lambda^{2}}{2} b(z) B_{f, \delta}(x)+O\left(\lambda^{3}\right) .
$$

Далее будем считать, что аддитивная помеха имеет стандартное нормальное распределение $\mathcal{N}(0 ; 1)$, для которого $g(z)=\phi(z)=\frac{1}{\sqrt{2 \pi}} e^{-\frac{x^{2}}{2}}$.

Отметим, что в области различения сигналов на фоне шумов рассматриваются не только гауссовские шумы. В качестве иллюстрации приведем ссылки на $[18,19]$, в которых рассматривались шумы с распределением Коши и бета-распределением.

Следствие 1. Для стандартного гауссовского шума с плотностью $\phi(z)$ имеет место формула

$$
r_{f, \lambda, \delta}(x, z)=1+\lambda z A_{f, \delta}(x)+\frac{\lambda^{2}}{2}\left(z^{2}-1\right) B_{f, \delta}(x)+O\left(\lambda^{3}\right) .
$$

Доказательство. Формула (6) следует непосредственно из (5) с учетом (4), а соотношение (5) вытекает из (3), (4) и равенств $\phi^{\prime}(z)=-z \phi(z)$, $\phi^{\prime \prime}(z)=\left(z^{2}-1\right) \phi(z)$, так что $a(z)=-z, b(z)=z^{2}-1$. 


\section{3. Асимптотически оптимальные критерии различе- ния гипотез $\boldsymbol{H}_{f, \delta}$ и $\boldsymbol{H}_{f^{*}, \delta^{*}}$ при $\delta=$ const, $\boldsymbol{\delta}^{*}=$ const, $\lambda \rightarrow \mathbf{0}$}

Пусть $n \rightarrow \infty, \lambda \equiv \lambda(n) \rightarrow 0, \delta=\left(\delta_{1}, \delta_{2}, \ldots, \delta_{m}\right)=$ const.

Построим асимптотически оптимальные критерии различения гипотез общего вида $H_{f, \delta}$ и $H_{f^{*}, \delta^{*}}$ по последовательности наблюдаемых векторов (2), а также найдем асимптотически минимальный объем выборки для различения гипотез при заданных вероятностях ошибок первого и второго рода.

Из леммы 1 непосредственно получаем следующее утверждение.

Лемма 2. Пусть $n \rightarrow \infty, \lambda=\lambda(n) \rightarrow 0$ и $l_{f, \lambda, \delta}(x, z)=\ln r_{f, \lambda, \delta}(x, z)$. Тогда справедливы следующие представления:

$$
\begin{gathered}
r_{f, \lambda, \delta}(x, z)=1+\lambda z A_{f, \delta}(x)+\frac{\lambda^{2}}{2}\left(z^{2}-1\right) B_{f, \delta}(x)+O\left(\lambda^{3}\right), \\
l_{f, \lambda, \delta}(x, z)=\lambda z A_{f, \delta}(x)+\frac{\lambda^{2}}{2}\left[\left(z^{2}-1\right) B_{f, \delta}(x)-z^{2} A_{f, \delta}^{2}(x)\right]+O\left(\lambda^{3}\right), \\
l_{f, f^{*}, \lambda, \lambda^{*}, \delta, \delta^{*}}(x, z)=\ln \frac{p_{f^{*}, \lambda^{*}, \delta^{*}}(x, z)}{p_{f, \lambda, \delta}(x, z)}=l_{f^{*}, \lambda^{*}, \delta^{*}}(x, z)-l_{f, \lambda, \delta}(x, z), \\
L_{f, f^{*}, \lambda, \lambda^{*}, \delta, \delta^{*}}\left(\left\{\left(X^{(t)}, \eta_{t}\right)\right\}_{t=1}^{n}\right)=\sum_{t=1}^{n} l_{f, f^{*}, \lambda, \lambda^{*}, \delta, \delta^{*}}\left(X^{(t)}, \eta_{t}\right) .
\end{gathered}
$$

Вначале предположим, что $f \equiv f^{*}$, и рассмотрим асимптотически оптимальные критерии различения гипотез $H_{\delta}$ и $H_{\delta^{*}}$. Через $\mathbf{E}^{(0)}$ ( ) далее обозначается математическое ожидание при гипотезе $H^{(0)}$.

Везде в дальнейшем символом « $\Rightarrow »$ будет обозначаться слабая сходимость вероятностных мер (или распределений).

Пусть $\delta=$ const, $\delta^{*}=$ const, $\delta \neq \delta^{*}$. Положим

$$
\begin{gathered}
L_{\delta, \delta^{*}} \equiv L_{\delta, \delta^{*}}\left(\left\{\left(X^{(t)}, \eta_{t}\right)\right\}_{t=1}^{n}\right)=\sum_{t=1}^{n} l_{\delta, \delta^{*}}\left(X^{(t)}, \eta_{t}\right)= \\
=\sum_{t=1}^{n}\left(l_{\delta^{*}}\left(X^{(t)}, \eta_{t}\right)-l_{\delta}\left(X^{(t)}, \eta_{t}\right)\right) .
\end{gathered}
$$

Tеорема 1. Пусть $n \rightarrow \infty, \lambda \equiv \lambda(n) \rightarrow 0, n \lambda^{2}=\gamma=$ const, $\delta=$ const, $\delta^{*}=\mathrm{const}, \quad \delta \neq \delta^{*}$. Положим $b\left(\delta, \delta^{*}\right)=\mathbf{E}^{(0)}\left(A_{\delta^{*}}\left(X^{(t)}\right)-A_{\delta}\left(X^{(t)}\right)\right)^{2}$. 
Тогда при гипотезе $H_{\delta}$

$$
\mathcal{L}_{\delta}\left(L_{\delta, \delta^{*}}\right) \Rightarrow \mathcal{N}\left(-\frac{\gamma}{2} b\left(\delta, \delta^{*}\right), \gamma b\left(\delta, \delta^{*}\right)\right)
$$

nри гипотезе $H_{\delta^{*}}$

$$
\mathcal{L}_{\delta^{*}}\left(L_{\delta, \delta^{*}}\right) \Rightarrow \mathcal{N}\left(\frac{\gamma}{2} b\left(\delta, \delta^{*}\right), \gamma b\left(\delta, \delta^{*}\right)\right) .
$$

Асимптотически минимальный объем выборки, достаточный для различения гипотез $H_{\delta}, H_{\delta^{*}}$ с вероятностями ошибок первого и второго рода $\alpha, \beta$ соответственно, равен

$$
n(\alpha, \beta) \sim \frac{\left(\zeta_{\alpha}+\zeta_{\beta}\right)^{2}}{\lambda^{2} b\left(\delta, \delta^{*}\right)} .
$$

Доказательство. Прежде всего напомним (см. (7)), что

$$
l_{\delta, \delta^{*}}(x, z)=l_{\delta^{*}}(x, z)-l_{\delta}(x, z) .
$$

Непосредственно устанавливается, что

$$
\mathbf{E}_{\delta} l_{\delta}\left(X^{(t)}, \eta_{t}\right)=\mathbf{E}^{(0)}\left[l_{\delta}\left(X^{(t)}, \eta_{t}\right) r_{\delta}\left(X^{(t)}, \eta_{t}\right)\right] .
$$

Из приведенных соотношений следует, что моменты логарифма отношения правдоподобия при гипотезах $H_{\delta}$ и $H_{\delta^{*}}$ могут быть представлены в виде

$$
\begin{aligned}
\mathbf{E}_{\delta} l_{\delta, \delta^{*}}\left(X^{(t)}, \eta_{t}\right) & =\mathbf{E}^{(0)}\left[\left(l_{\delta^{*}}\left(X^{(t)}, \eta_{t}\right)-l_{\delta}\left(X^{(t)}, \eta_{t}\right)\right) r_{\delta}\left(X^{(t)}, \eta_{t}\right)\right], \\
\mathbf{E}_{\delta^{*}} l_{\delta, \delta^{*}}\left(X^{(t)}, \eta_{t}\right) & =\mathbf{E}^{(0)}\left[\left(l_{\delta^{*}}\left(X^{(t)}, \eta_{t}\right)-l_{\delta}\left(X^{(t)}, \eta_{t}\right)\right) r_{\delta^{*}}\left(X^{(t)}, \eta_{t}\right)\right] .
\end{aligned}
$$

Проводя соответствующие вычисления, с учетом (6), (7) в условиях теоремы получаем

$$
\begin{gathered}
\mathbf{E}_{\delta} l_{\delta, \delta^{*}}\left(X^{(t)}, \eta_{t}\right)=\mathbf{E}^{(0)}\left[l_{\delta, \delta^{*}}\left(X^{(t)}, \eta_{t}\right) r_{\delta}\left(X^{(t)}, \eta_{t}\right)\right]= \\
=\mathbf{E}^{(0)}\left[\left(\lambda \eta_{t}\left(A_{\delta^{*}}\left(X^{(t)}\right)-A_{\delta}\left(X^{(t)}\right)\right)+\right.\right. \\
\left.+\frac{\lambda^{2}}{2}\left[\left(\eta_{t}^{2}-1\right) B_{\delta}\left(X^{(t)}\right)-\eta_{t}^{2}\left(A_{\delta^{*}}^{2}\left(X^{(t)}\right)-A_{\delta}^{2}\left(X^{(t)}\right)\right)\right]+O\left(\lambda^{3}\right)\right) \times \\
\left.\times\left(1+\lambda \eta_{t} A_{\delta}\left(X^{(t)}\right)+\frac{\lambda^{2}}{2}\left(\eta_{t}^{2}-1\right) B_{\delta}\left(X^{(t)}\right)+O\left(\lambda^{3}\right)\right)\right]= \\
=-\frac{\lambda^{2}}{2} \mathbf{E}^{(0)}\left(A_{\delta^{*}}\left(X^{(t)}\right)-A_{\delta}\left(X^{(t)}\right)\right)^{2} .
\end{gathered}
$$


Аналогично устанавливается, что

$$
\begin{gathered}
\mathbf{E}_{\delta^{*}} l_{\delta, \delta^{*}}\left(X^{(t)}, \eta_{t}\right)=\mathbf{E}^{(0)}\left[l_{\delta, \delta^{*}}\left(X^{(t)}, \eta_{t}\right) r_{\delta^{*}}\left(X^{(t)}, \eta_{t}\right)\right]= \\
=\frac{\lambda^{2}}{2} \mathbf{E}^{(0)}\left(A_{\delta^{*}}\left(X^{(t)}\right)-A_{\delta}\left(X^{(t)}\right)\right)^{2} .
\end{gathered}
$$

Из (11), (12) следует, что

$$
\begin{gathered}
\mathbf{E}_{\delta^{*}} l_{\delta, \delta^{*}}\left(X^{(t)}, \eta_{t}\right)=\mathbf{E}^{(0)}\left[\left(l_{\delta^{*}}\left(X^{(t)}, \eta_{t}\right)-l_{\delta}\left(X^{(t)}, \eta_{t}\right)\right) r_{\delta^{*}}\left(X^{(t)}, \eta_{t}\right)\right]= \\
=\frac{\lambda^{2}}{2} \mathbf{E}^{(0)}\left(A_{\delta^{*}}\left(X^{(t)}\right)-A_{\delta}\left(X^{(t)}\right)\right)^{2}+O\left(\lambda^{3}\right) .
\end{gathered}
$$

Аналогично устанавливается, что

$$
\begin{gathered}
\mathbf{E}_{\delta} l_{\delta, \delta^{*}}\left(X^{(t)}, \eta_{t}\right)=\mathbf{E}^{(0)}\left[l_{\delta, \delta^{*}}\left(X^{(t)}, \eta_{t}\right) r_{\delta}\left(X^{(t)}, \eta_{t}\right)\right]= \\
=-\frac{\lambda^{2}}{2} \mathbf{E}^{(0)}\left(A_{\delta^{*}}\left(X^{(t)}\right)-A_{\delta}\left(X^{(t)}\right)\right)^{2}+O\left(\lambda^{3}\right) .
\end{gathered}
$$

По аналогии с доказательством теоремы 1 в [10] с учетом того, что $b\left(\delta, \delta^{*}\right)=\mathbf{E}^{(0)}\left(A_{\delta^{*}}\left(X^{(t)}\right)-A_{\delta}\left(X^{(t)}\right)\right)^{2}$, а также представлений (13), (14) можно сделать вывод о том, что при справедливости гипотезы $H_{\delta}$ распределение статистики логарифма отношения правдоподобия $L_{\delta, \delta^{*}}$ в условиях теоремы слабо сходится к нормальному распределению $\mathcal{N}\left(-\frac{\gamma^{2}}{2} b\left(\delta, \delta^{*}\right), \gamma^{2} b\left(\delta, \delta^{*}\right)\right)$, а при справедливости гипотезы $H_{\delta^{*}}-$ к нормальному распределению $\mathcal{N}\left(\frac{\gamma^{2}}{2} b\left(\delta, \delta^{*}\right), \gamma^{2} b\left(\delta, \delta^{*}\right)\right)$. Тем самым доказаны утверждения (8), (9).

Для доказательства (10) заметим, что для границ оптимального критерия с учетом асимптотической нормальности статистики логарифма отношения правдоподобия верны предельные соотношения

$$
-\frac{\gamma^{2}}{2} b\left(\delta, \delta^{*}\right)-\varsigma_{\alpha} \sqrt{\gamma^{2} b\left(\delta, \delta^{*}\right)} \sim \frac{\gamma^{2}}{2} b\left(\delta, \delta^{*}\right)+\varsigma_{\beta} \sqrt{\gamma^{2} b\left(\delta, \delta^{*}\right)},
$$

откуда следует асимптотическое равенство

$$
\left(\varsigma_{\alpha}+\varsigma_{\beta}\right)^{2} \sim \gamma^{2} b\left(\delta, \delta^{*}\right)=n \lambda^{2} b\left(\delta, \delta^{*}\right) .
$$

Из приведенных рассуждений следует, что асимптотически минимальный объем $n(\alpha, \beta)$ выборки, достаточный для различения гипотез $H_{\delta}, H_{\delta^{*}}$ с заданными вероятностями ошибок первого и второго рода $\alpha, \beta$ соответственно, удовлетворяет соотношению $n(\alpha, \beta) \sim \frac{\left(\varsigma_{\alpha}+\varsigma_{\beta}\right)^{2}}{\lambda^{2} b\left(\delta, \delta^{*}\right)}, \lambda \rightarrow 0$. 
Рассмотрим важный для приложений частный случай $f(x)=\|x\|=$ $=\sum_{j=1}^{m} x_{j}$. С учетом того, что

$$
\mathbf{E}_{0} \varepsilon_{j}=\frac{1}{2}, \quad \mathbf{E}_{0}(-1)^{\varepsilon_{j}}=0, \quad \mathbf{E}_{0}\left[\varepsilon_{j}(-1)^{\varepsilon_{j}}\right]=-\frac{1}{2},
$$

нетрудно получить следующие соотношения:

$$
\begin{gathered}
A_{\delta}(x)=\mathbf{E}_{\delta} f(x \oplus \varepsilon)=\mathbf{E}_{0}\left[f(x \oplus \varepsilon) \frac{\pi_{\delta}(\varepsilon)}{\pi_{0}(\varepsilon)}\right]= \\
=\mathbf{E}_{0}\left[f(x \oplus \varepsilon) \prod_{j=1}^{m}\left(1+(-1)^{\varepsilon_{j}} \delta_{j}\right)\right]= \\
=\mathbf{E}_{0}\left[\sum_{j=1}^{m}\left(\varepsilon_{j}+(-1)^{\varepsilon_{j}} x_{j}\right) \prod_{j=1}^{m}\left(1+(-1)^{\varepsilon_{j}} \delta_{j}\right)\right]= \\
\left.\sum_{j=1}^{m}\left(\begin{array}{c}
\left.\left.\mathbf{E}_{0}\left[\left(\varepsilon_{j}+(-1)^{\varepsilon_{j}} x_{j}\right)\left(1+(-1)^{\varepsilon_{j}} \delta_{j}\right)\right] \mathbf{E}_{0}\left[\begin{array}{l}
m \\
i \neq j
\end{array}\right]+(-1)^{\varepsilon_{i}} \delta_{i}\right)\right] \\
i, j=1
\end{array}\right]\right) \\
=\frac{m}{2}+\sum_{j=1}^{m} \delta_{j}\left(x_{j}-\frac{1}{2}\right) .
\end{gathered}
$$

Отсюда следует, что

$$
\begin{gathered}
\mathbf{E}^{(0)}\left(A_{\delta^{*}}\left(X^{(t)}\right)-A_{\delta}\left(X^{(t)}\right)\right)^{2}=\mathbf{E}^{(0)}\left(\sum_{j=1}^{m}\left(\delta_{j}^{*}-\delta_{j}\right)\left(X_{j}^{(t)}-\frac{1}{2}\right)\right)^{2}= \\
=\frac{1}{4} \sum_{j=1}^{m}\left(\delta_{j}^{*}-\delta_{j}\right)^{2}=\frac{1}{4} d\left(\delta, \delta^{*}\right),
\end{gathered}
$$

где $d\left(\delta, \delta^{*}\right)$ - квадрат расстояния между векторами $\delta$ и $\delta^{*}$ :

$$
d\left(\delta, \delta^{*}\right)=\sum_{j=1}^{m}\left(\delta_{j}^{*}-\delta_{j}\right)^{2}
$$


А. В. Иванов

Таким образом, асимптотически минимальный объем выборки, достаточный для различения гипотез $H_{\delta}, H_{\delta^{*}}$ с заданными вероятностями ошибок первого и второго рода $\alpha, \beta$ соответственно, с учетом (10) асимптотически равен

$$
n(\alpha, \beta) \sim \frac{4\left(\zeta_{\alpha}+\zeta_{\beta}\right)^{2}}{\lambda^{2} d\left(\delta, \delta^{*}\right)} .
$$

Полученный результат сформулируем в виде следующего утверждения.

Следствие 2. Пусть $n \rightarrow \infty, \lambda \equiv \lambda(n) \rightarrow 0, n \lambda^{2}=\gamma=$ const, $\delta=\left(\delta_{1}, \delta_{2}, \ldots, \delta_{m}\right)=$ const, $\delta^{*}=\left(\delta_{1}^{*}, \delta_{2}^{*}, \ldots, \delta_{m}^{*}\right)=$ const, $\delta \neq \delta^{*}, f(x)=$ $=\|x\|=\sum_{j=1}^{m} x_{j}$ и $d\left(\delta, \delta^{*}\right)=\sum_{j=1}^{m}\left(\delta_{j}^{*}-\delta_{j}\right)^{2}$. Тогда асимптотически минимальный объем выборки, достаточный для различения гипотез $H_{\delta}, H_{\delta^{*}}$ с вероятностями ошибок первого и второго рода $\alpha$, $\beta$ соответственно, равен

$$
n(\alpha, \beta) \sim \frac{4\left(\varsigma_{\alpha}+\varsigma_{\beta}\right)^{2}}{\lambda^{2} d\left(\delta, \delta^{*}\right)} .
$$

В частном случае, когда $\delta_{j}=\delta, \delta_{j}^{*}=\delta^{*}, j=1,2, \ldots, m, \delta \neq \delta^{*}$, получаем следующее утверждение.

Следствие 3. Пусть $n \rightarrow \infty, \lambda \equiv \lambda(n) \rightarrow 0, n \lambda^{2}=\gamma=$ const, $f(x)=$ $=\|x\|=\sum_{j=1}^{m} x_{j}$ u $\delta_{j}=\delta=$ const, $\delta_{j}^{*}=\delta^{*}=$ const, $\delta \neq \delta^{*}$. Тогда acumnmoтически минимальный объем выборки, достаточный для различения гипотез $H_{\delta}$ и $H_{\delta^{*}}$ с вероятностями ошибок первого и второго рода $\alpha, \beta$ соответственно, равен

$$
n(\alpha, \beta) \sim \frac{4\left(\zeta_{\alpha}+\zeta_{\beta}\right)^{2}}{\lambda^{2} m\left(\delta-\delta^{*}\right)^{2}} .
$$

В частности, из следствия 3 при $\delta=1, \delta^{*}=0$ следует теорема 1 в $[6$, с. 95$]$.

\section{4. Асимптотически оптимальные критерии различения гипотез $\boldsymbol{H}_{f, \delta}$ и $\boldsymbol{H}_{f^{*}, \delta^{*}}$

$$
\text { при } \delta=\delta(n)=\left(\delta_{1}(n), \ldots, \delta_{m}(n)\right) \rightarrow 0, \lambda \rightarrow 0
$$

Теперь рассмотрим случай, когда $n \rightarrow \infty, \lambda \equiv \lambda(n) \rightarrow 0$ и $\delta=\delta(n)=$ $=\left(\delta_{1}(n), \ldots, \delta_{m}(n)\right) \rightarrow 0$. Для построения критерия найдем разложение отношения плотностей $r_{f, \lambda, \delta}(x, z)$ (3) в этих условиях с использованием формулы Тейлора для функций от нескольких переменных (см., например, [11, п. 4.10-5, с. 142]). 
При фиксированном векторе $u$ временно введем обозначение $\pi(\delta)=$ $=\pi_{\delta}(u)$. Тогда в окрестности вектора $a=\left(a_{1}, \ldots, a_{m}\right)$ имеет место формула

$$
\begin{gathered}
\pi(\delta)=\pi\left(\delta_{1}, \ldots, \delta_{m}\right)=\pi\left(a_{1}, \ldots, a_{m}\right)+\left.\sum_{i=1}^{m} \frac{\partial \pi}{\partial \delta_{i}}\right|_{\left(a_{1}, \ldots, a_{m}\right)}\left(\delta_{i}-a_{i}\right)+ \\
+\left.\frac{1}{2} \sum_{i=1}^{m} \sum_{j=1}^{m} \frac{\partial^{2} \pi}{\partial \delta_{i} \partial \delta_{j}}\right|_{\left(a_{1}, \ldots, a_{m}\right)}\left(\delta_{i}-a_{i}\right)\left(\delta_{j}-a_{j}\right)+ \\
+\left.\frac{1}{6} \sum_{i=1}^{m} \sum_{j=1}^{m} \sum_{k=1}^{m} \frac{\partial^{3} \pi}{\partial \delta_{i} \partial \delta_{j} \partial \delta_{k}}\right|_{\left(a_{1}, \ldots, a_{m}\right)}\left(\delta_{i}-a_{i}\right)\left(\delta_{j}-a_{j}\right)\left(\delta_{k}-a_{k}\right)+R_{4},
\end{gathered}
$$

или, в общем случае,

$$
\pi(\delta)=\pi\left(\delta_{1}, \ldots, \delta_{m}\right)=\pi(a)+d \pi+\frac{1}{2} d^{2} \pi+\ldots+\frac{1}{(k-1) !} d^{k-1} \pi+R_{k},
$$

где

$$
\begin{gathered}
\left.d^{k} \pi\right|_{\left(a_{1}, \ldots, a_{m}\right)}=\left(\frac{\partial \pi(\delta)}{\partial \delta_{1}} d \delta_{1}+\ldots+\frac{\partial \pi(\delta)}{\partial \delta_{m}} d \delta_{m}\right)^{k} \pi\left(\delta_{1}, \ldots, \delta_{m}\right), \\
R_{k}=\frac{1}{k !} d^{k} \pi(\nu), \quad v=\left(v_{1}, \ldots, v_{m}\right), \quad v=a+\theta(\delta-a), 0<\theta<1 .
\end{gathered}
$$

Перейдем к матричным обозначениям. Пусть $\dot{\pi}_{0}(u)=$ $=\left(\left.\frac{\partial \pi(\delta)}{\partial \delta_{1}}\right|_{\delta=0}, \ldots,\left.\frac{\partial \pi(\delta)}{\partial \delta_{m}}\right|_{\delta=0}\right)^{T}-$ вектор-столбец, $T$ - операция транспонирования, $\ddot{\pi}_{0}(u)=\left\|\left.\frac{\partial^{2} \pi(\delta)}{\partial \delta_{i} \partial \delta_{j}}\right|_{\delta=0}\right\|_{i, j=1}^{m}-$ квадратная матрица,

$$
\begin{aligned}
h(u) & =\frac{\dot{\pi}_{0}(u)}{\pi_{0}(u)}, & h_{\delta}(u) & =\delta h(u), \\
H(u) & =\frac{\ddot{\pi}_{0}(u)}{\pi_{0}(u)}, & H_{\delta}(u) & =\delta H(u) \delta^{T} .
\end{aligned}
$$

Тогда разложение функции $\pi_{\delta}(u)$ в ряд Тейлора по малому параметру $\delta$ может быть записано в виде

$$
\begin{gathered}
\pi_{\delta}(u)=\pi_{0}(u)+\delta \dot{\pi}_{0}(u)+\frac{1}{2} \delta \ddot{\pi}_{0}(u) \delta^{T}+R_{3}= \\
=\pi_{0}(u)\left(1+h_{\delta}(u)+\frac{1}{2} H_{\delta}(u)+R_{3}\right),
\end{gathered}
$$


откуда следуют соотношения

$$
\begin{gathered}
A_{\delta}(x)=\mathbf{E}_{\delta} f(x \oplus \varepsilon)=\mathbf{E}_{0}\left[f(x \oplus \varepsilon) \pi_{\delta}(\varepsilon) \pi_{0}^{-1}(\varepsilon)\right]= \\
=\mathbf{E}_{0}\left[f(x \oplus \varepsilon)\left(1+h_{\delta}(\varepsilon)+\frac{1}{2} H_{\delta}(\varepsilon)+R_{3}\right)\right] .
\end{gathered}
$$

Следовательно, учитывая представление (3), получаем:

$$
\begin{aligned}
r_{f, \lambda, \delta}(x, z) & =1+\lambda z A_{\delta}(x)+\frac{\lambda^{2}}{2}\left(z^{2}-1\right) B_{\delta}(x)+O\left(\lambda^{3}\right)= \\
& =1+\lambda z \mathbf{E}_{0}\left[f(x \oplus \varepsilon) \pi_{\delta}(\varepsilon) \pi_{0}^{-1}(\varepsilon)\right]+ \\
+ & \frac{\lambda^{2}}{2}\left(z^{2}-1\right) \mathbf{E}_{0}\left[f^{2}(x \oplus \varepsilon) \pi_{\delta}(\varepsilon) \pi_{0}^{-1}(\varepsilon)\right]+O\left(\lambda^{3}\right) .
\end{aligned}
$$

Лемма 3. Пусть $h_{\delta}(u)=\delta \frac{\dot{\pi}_{0}(u)}{\pi_{0}(u)}, H_{\delta}(u)=\delta \frac{\ddot{\pi}_{0}(u)}{\pi_{0}(u)} \delta^{T}, R_{3}$ определеньл в (17), (18), $A_{\delta}(x)=\mathbf{E}_{\delta} f(x \oplus \varepsilon), B_{\delta}(x)=\mathbf{E}_{\delta} f^{2}(x \oplus \varepsilon)$. Тогда

$$
\begin{gathered}
r_{f, \lambda, \delta}(x, z)=1+\lambda z \mathbf{E}_{0}\left[f(x \oplus \varepsilon)\left(1+h_{\delta}(\varepsilon)+\frac{1}{2} H_{\delta}(\varepsilon)+R_{3}\right)\right]+ \\
+\frac{\lambda^{2}}{2}\left(z^{2}-1\right) \mathbf{E}_{0}\left[f^{2}(x \oplus \varepsilon)\left(1+h_{\delta}(\varepsilon)+\frac{1}{2} H_{\delta}(\varepsilon)+R_{3}\right)\right]+O\left(\lambda^{3}\right) .
\end{gathered}
$$

Обозначим длину вектора $\delta$ через $\theta=\|\delta\|=\sqrt{\sum_{j=1}^{m} \delta_{j}^{2}}$. Положим $\chi=$ $=\delta \theta^{-1}$. Тогда $\delta=\theta \chi$ и при $\delta \rightarrow 0$ вектор $\chi=$ const определяет «направление» сближения вектора $\delta$ с началом координат, а величина $\theta$ - «скорость» сближения. Через $H_{\chi}$ далее обозначим гипотезу, при которой $\theta \rightarrow 0$ и $\chi=$ $=$ const. В этом случае из (19) следует представление

$$
\begin{gathered}
\pi_{\delta}(u)=\pi_{\varepsilon \chi}(u)=\pi_{0}(u)+\varepsilon \chi \dot{\pi}_{0}(u)+\left(\frac{\varepsilon^{2}}{2}\right) \chi \ddot{\pi}_{0}(u) \chi^{T}+O\left(\varepsilon^{3}\right)= \\
=\pi_{0}\left(1+\varepsilon h_{\chi}(u)+\frac{\varepsilon^{2}}{2} H_{\chi}(u)+O\left(\varepsilon^{3}\right)\right) .
\end{gathered}
$$

Лемма 4.

1. Пусть $\lambda \rightarrow 0, \delta=\theta \chi, \theta \rightarrow 0, \chi=$ const. Тогдa

$$
\begin{gathered}
r_{\delta}(x, z)=1+\lambda z \mathbf{E}_{0} f(x \oplus \varepsilon)+ \\
+\lambda \theta z \mathbf{E}_{0}\left[f(x \oplus \varepsilon)\left(h_{\chi}(\varepsilon)+\frac{\theta}{2} H_{\chi}(\varepsilon)+O\left(\theta^{2}\right)\right)\right]+
\end{gathered}
$$




$$
\begin{gathered}
+\frac{\lambda^{2}}{2}\left(z^{2}-1\right) \mathbf{E}_{0} f^{2}(x \oplus \varepsilon)+ \\
+\frac{\lambda^{2}}{2} \theta\left(z^{2}-1\right) \mathbf{E}_{0}\left[f^{2}(x \oplus \varepsilon)\left(h_{\chi}(\varepsilon)+\frac{\theta}{2} H_{\chi}(\varepsilon)+O\left(\theta^{2}\right)\right)\right]+O\left(\lambda^{3}\right) .
\end{gathered}
$$

2. Пусmb $\lambda \rightarrow 0, \delta=\theta \chi, \theta=\rho \lambda(\delta=\rho \lambda \chi), \rho=$ const, $\chi=$ const. Тогда

$$
\begin{gathered}
r_{\delta}(x, z)=1+\lambda z \mathbf{E}_{0} f(x \oplus \varepsilon)+ \\
+\lambda^{2}\left[z \rho \mathbf{E}_{0}\left[f(x \oplus \varepsilon) h_{\chi}(\varepsilon)\right]+\frac{1}{2}\left(z^{2}-1\right) \mathbf{E}_{0} f^{2}(x \oplus \varepsilon)\right]+ \\
+\frac{\lambda^{3}}{2}\left[\rho\left(z^{2}-1\right) \mathbf{E}_{0}\left[f^{2}(x \oplus \varepsilon) h_{\chi}(\varepsilon)\right]+\right. \\
\left.+z \rho^{2} \mathbf{E}_{0}\left[f(x \oplus \varepsilon) H_{\chi}(\varepsilon)\right]\right]+O\left(\lambda^{4}\right) .
\end{gathered}
$$

Доказательство утверждений (21), (22) можно получить, подставив конкретный вид параметра $\theta$ в представление (20) и сгруппировав коэффициенты при одинаковых степенях параметра $\lambda$.

Теорема 2. Пусть $n \rightarrow \infty, \lambda=\lambda(n) \rightarrow 0, n \lambda^{4}=\gamma=$ const, $\delta \equiv$ $\equiv \delta(n)=\rho \lambda \chi, \delta^{*} \equiv \delta^{*}(n)=\rho^{*} \lambda \chi, \rho=$ const, $\rho^{*}=$ const, $\rho \neq \rho^{*}$. Пycmb

$$
q_{\chi}(x)=\mathbf{E}^{(0)}\left[f(x \oplus \varepsilon) h_{\chi}(\varepsilon)\right], Q_{\chi}=\mathbf{E}^{(0)} q_{\chi}^{2}\left(X^{(t)}\right) .
$$

Тогда при гипотезе $H_{\delta}$

$$
\mathcal{L}_{\delta}\left(L_{\delta, \delta^{*}}\right) \Rightarrow \mathcal{N}\left(-\frac{\gamma\left(\rho^{*}-\rho\right)^{2} Q_{\chi}}{2}, \gamma\left(\rho^{*}-\rho\right)^{2} Q_{\chi}\right)
$$

при гипотезе $H_{\delta^{*}}$

$$
\mathcal{L}_{\delta^{*}}\left(L_{\delta, \delta^{*}}\right) \Rightarrow \mathcal{N}\left(\frac{\gamma\left(\rho^{*}-\rho\right)^{2} Q_{\chi}}{2}, \gamma\left(\rho^{*}-\rho\right)^{2} Q_{\chi}\right)
$$

Асимптотически минимальный объем выборки для различения гипотез $H_{\delta}$, $H_{\delta^{*}}$ с вероятностями ошибок первого и второго рода $\alpha$, $\beta$ соответственно равен

$$
n(\alpha, \beta) \sim \frac{\left(u_{\alpha}+u_{\beta}\right)^{2}}{\lambda^{4}\left(\rho^{*}-\rho\right)^{2} Q_{\chi}} .
$$


Доказательство. Найдем математическое ожидание и дисперсию $L_{\delta, \delta^{*}}\left(\left\{\left(X^{(t)}, \eta_{t}\right)\right\}_{t=1}^{n}\right)=\sum_{t=1}^{n} l_{\delta, \delta^{*}}\left(X^{(t)}, \eta_{t}\right)$. Заметим, что для математического ожидания $\mathbf{E}_{\tau} l_{\delta, \delta^{*}}\left(X^{(t)}, \eta_{t}\right)$ верно представление

$$
\mathbf{E}_{\tau} l_{\delta, \delta^{*}}\left(X^{(t)}, \eta_{t}\right)=\mathbf{E}^{(0)}\left(l_{\delta, \delta^{*}}\left(X^{(t)}, \eta_{t}\right) r_{\tau}\left(X^{(t)}, \eta_{t}\right)\right)
$$

где $\tau \in\left\{\delta, \delta^{*}\right\}$. Проведя вычисление коэффициентов в представлении (21) при степенях $\lambda$ до четвертого порядка включительно и учитывая, что

$$
l_{\delta, \delta^{*}}(x, z)=l_{\delta^{*}}(x, z)-l_{\delta}(x, z)=\ln \frac{r_{\delta^{*}}(x, z)}{r_{\delta}(x, z)},
$$

устанавливаем, что

$$
\mathbf{E}_{\delta} l_{\delta, \delta^{*}}\left(X^{(t)}, \eta_{t}\right) \sim-\frac{\lambda^{4}\left(\rho^{*}-\rho\right)^{2} \mathbf{E}^{(0)} q_{\chi}^{2}\left(X^{(t)}\right)}{2}=-\frac{\lambda^{4}\left(\rho^{*}-\rho\right)^{2} Q_{\chi}}{2} .
$$

Аналогично убеждаемся, что

$$
\mathbf{E}_{\delta^{*}} l_{\delta, \delta^{*}}\left(X^{(t)}, \eta_{t}\right) \sim \frac{\lambda^{4}\left(\rho^{*}-\rho\right)^{2} Q_{\chi}}{2}
$$

и

$$
\mathbf{D}_{\delta} l_{\delta, \delta^{*}}\left(X^{(t)}, \eta_{t}\right) \sim \mathbf{D}_{\delta^{*}} l_{\delta, \delta^{*}}\left(X^{(t)}, \eta_{t}\right) \sim \lambda^{4}\left(\rho^{*}-\rho\right)^{2} Q_{\chi}
$$

Принимая во внимание асимптотическую нормальность статистики $L_{\delta, \delta^{*}}$, которая устанавливается аналогично тому, как это было сделано в [10], и учитывая, что

$$
L_{\delta, \delta^{*}}\left(\left\{\left(X^{(t)}, \eta_{t}\right)\right\}_{t=1}^{n}\right)=\sum_{t=1}^{n} l_{\delta, \delta^{*}}\left(X^{(t)}, \eta_{t}\right)
$$

заключаем, что при гипотезе $H_{\delta}$ выполнено предельное соотношение

$$
L_{\delta, \delta^{*}} \sim \mathcal{N}\left(-\frac{\gamma\left(\rho^{*}-\rho\right)^{2} Q_{\chi}}{2}, \gamma\left(\rho^{*}-\rho\right)^{2} Q_{\chi}\right)
$$

а при гипотезе $H_{\delta^{*}}-$ соотношение

$$
L_{\delta, \delta^{*}} \sim \mathcal{N}\left(\frac{\gamma\left(\rho^{*}-\rho\right)^{2} Q_{\chi}}{2}, \gamma\left(\rho^{*}-\rho\right)^{2} Q_{\chi}\right)
$$


Тем самым утверждения (23), (24) доказаны. Из (26), (27) стандартными преобразованиями получаем приближенное равенство

$$
n\left(\rho^{*}-\rho\right)^{2} \lambda^{4} Q_{\chi} \sim\left(\varsigma_{\alpha}+\varsigma_{\beta}\right)^{2},
$$

откуда следует (25)

$$
n(\alpha, \beta) \sim \frac{\left(\varsigma_{\alpha}+\varsigma_{\beta}\right)^{2}}{\lambda^{4}\left(\rho^{*}-\rho\right)^{2} Q_{\chi}} .
$$

Заметим, что при $\delta_{j}=\delta, j=1,2, \ldots, m, \chi=(1,1, \ldots, 1)$ приходим к оптимальному критерию различения гипотез $H_{\delta}: \delta=\rho \lambda$ и $H_{\delta^{*}}: \delta=$ $=\rho^{*} \lambda$, изучавшемуся ранее в [8], и в качестве следствий теоремы 2 получаем утверждения теоремы 2 в [8].

\section{Выводы}

1. Как показали проведенные исследования, параметры предельных распределений логарифма статистики отношения правдоподобия критерия различения гипотез $H_{\delta}, H_{\delta^{*}}$ при $n \rightarrow \infty, \lambda \equiv \lambda(n) \rightarrow 0$ существенно зависят от вида векторного параметра $\delta$ и различаются при $\delta=$ const и $\delta \rightarrow 0$.

2. При различении гипотез $H_{\delta}, H_{\delta^{*}}$, где $\delta=$ const, $\delta^{*}=$ const, $\delta \neq \delta^{*}$, асимптотически минимальный объем выборки составляет величину порядка $O\left(\frac{1}{\lambda^{2}}\right)$, в то время как при $\delta=\rho \lambda \chi, \delta^{*}=\rho^{*} \lambda \chi, \rho=$ const $>0, \rho^{*}=$ $=$ const $>0, \rho \neq \rho^{*}-$ величину порядка $O\left(\frac{1}{\lambda^{4}}\right)$. Указанный эффект зависит от отсутствия или наличия взаимосвязей между параметрами $\delta, \delta^{*}$ в схеме серий.

3. В дальнейшем определенный интерес представляют построение асимптотически оптимальных критериев различения гипотез и расчет асимптотически минимального объема выборки в следующих случаях:

- при обобщении модели (1), когда вместо операции $\oplus$ используется любая другая операция (например, умножение или сложение по некоторому модулю);

- при рассмотрении модели (1), в которой в качестве непрерывной помехи присутствуют шумы с отличными от гауссовского распределениями;

- при $\delta=\rho \lambda \chi, \delta^{*}=\rho^{*} \lambda \chi^{*}$, когда убывание происходит по разным «направлениям» $\chi$ и $\chi^{*}$;

- при различении гипотез для параметров $\delta=\rho \lambda \chi$ и $\delta^{*}=0$;

- для случая зависимых векторов $\left\{\varepsilon^{(t)}\right\}_{t=1}^{\infty}$ (например, для $k$-зависимых случайных векторов). 
А. В. Иванов

\section{Список литературы}

[1] Арбеков И.М., “Оптимальная дискретизация наблюдений слабых сигналов при ограничении на скорость квантования", Проблемы передачи информации, 34:1 (1998), 69-76.

[2] Боровков А.А., Математическая статистика (4-е изд.), Санкт-ПетербургМосква-Краснодар: Лань, 2010, 704 с.

[3] Вернер М., Основы кодирования (сер. «Мир программирования»), М.: Техносфера, 2004, $288 \mathrm{c.}$

[4] Гаек Я., Шидак 3., Теория ранговых критериев, М.: Наука, 1971, 376 с.

[5] Гнеденко Б.В., Курс теории вероятностей (6-е изд.), М.: Наука, 1988, 448 с.

[6] Иванов А.В., “Асимптотически наиболее мощный критерий различения гипотез о распределении случайного вектора”, Десятая общеросс. научн. конф. «Математика и безопасность информационных технологий (МаБИТ-2011)». Материалы секции «Математические проблемы информационной безопасности», МГУ, 13 октября 2011 г., М.: МАКС-ПРЕСС, 2012, 93-97.

[7] Иванов А.В., “Асимптотически оптимальные критерии в задаче различения гипотез о распределении случайного вектора", Обозр. прикл. промышл. матем., 20:2 (2013), 139-141.

[8] Иванов А.В., “Асимптотически оптимальные критерии в задаче различения гипотез о распределении случайного вектора. II”, Обозр. прикл. промышл. матем., 20:4 (2013), 548-550.

[9] Иванов А.В., “Асимптотически оптимальные критерии в задаче различения гипотез о распределении случайного вектора. III”, Обозр. прикл. промышл. матем.,21:1 (2014), 58-60.

[10] Иванов А.В., “Асимптотически оптимальные критерии в задаче различения параметрических гипотез о распределении случайного вектора. I”, Математические вопросы криптографии, 6:3 (2015), 87-114.

[11] Корн Г., Корн Т., Справочник по математике, М.: Наука, 1968, 720 с.

[12] Пазизин С.В., “Обнаружение и прием последовательности сигналов, искаженных случайной помехой и независимым шумом”, Проблемы передачи информаичи, 34:1 (1998), 46-55.

[13] Пазизин С.В., "Вероятности правильного декодирования для канала с аддитивным нормальным шумом и двоичного симметричного канала при случайном выборе кодовых слов”, Дискретная математика, 12:2 (2000), 93-98.

[14] Петров В.В., "Предельные теоремы для сумм независимых случайных величин”, М.: Наука, 1987, 320 с.

[15] Русас Дж., Контигуальность вероятностных мер, М.: Мир, 1975, 256 с.

[16] Чибисов Д.М., “Теорема о допустимых критериях и ее применение к одной асимптотической задаче проверки гипотез”, Теория вероятностей и ее применения, XXI:1 (1967), 96-111.

[17] Arbekov I.M., "Asymptotically optimum detection of a weak signal sequence with random time delays", IEEE Trans. Inf. Theory, 41:4 (1995), 1169-1174.

[18] Kassam Saleem A., "Signal detection in non-Gaussian noise", New York: SpringerVerlag, 1988, $235 \mathrm{p}$.

[19] Miller James H., Thomas John B., "Detectors for discrete-time signals in nonGaussian noise", IEEE Transactions on Information Theory, 18:2 (1972), 241-250. 